Review Article

\title{
Repetitive Transcranial Magnetic Stimulation for Neuropathic Pain on the Non-Motor Cortex: An Evidence Mapping of Systematic Reviews
}

\author{
Yaning Zang $\left(\mathbb{D},{ }^{1}\right.$ Yongni Zhang $\left(\mathbb{D},{ }^{2}\right.$ Xigui Lai ${ }^{D},{ }^{1}$ Yujie Yang ${ }^{(D)},{ }^{3}$ Jiabao Guo $(\mathbb{D}, 4$ \\ Shanshan Gu $\oplus^{5},{ }^{5}$ and Yi Zhu ${ }^{6}$ \\ ${ }^{1}$ Department of Kinesiology, Shanghai University of Sport, Shanghai, China \\ ${ }^{2}$ School of Health Sciences, Duquesne University, Pittsburgh, PA, USA \\ ${ }^{3}$ Department of Biomedical Sciences, City University of Hong Kong, Kowloon, Hong Kong, China \\ ${ }^{4}$ Department of Rehabilitation Medicine, The Second School of Clinical Medicine, Xuzhou Medical University, Xuzhou, \\ Jiangsu, China \\ ${ }^{5}$ Department of Physical Therapy, University of Toronto, Toronto, ON, Canada \\ ${ }^{6}$ Department of Musculoskeletal Pain Rehabilitation, The Fifth Affiliated Hospital of Zhengzhou University, Zhengzhou, \\ Henan, China \\ Correspondence should be addressed to Yi Zhu; zhuyi1010@163.com
}

Received 28 July 2021; Revised 4 October 2021; Accepted 12 October 2021; Published 29 October 2021

Academic Editor: Feng Zhang

Copyright $\odot 2021$ Yaning Zang et al. This is an open access article distributed under the Creative Commons Attribution License, which permits unrestricted use, distribution, and reproduction in any medium, provided the original work is properly cited.

Objective. This study was aimed to summarize and analyze the quality of the available evidence in systematic reviews (SRs) of repetitive transcranial magnetic stimulation (rTMS) on the non-motor cortex (non-M1) for neuropathic pain (NP) through an evidence mapping approach. Methods. We follow the Global Evidence Mapping (GEM) methodology. Searches were conducted in PubMed, EMBASE, Epistemonikos, and the Cochrane Library. The study type was restricted to SRs with or without meta-analysis. All literature published before January 23, 2021, were included. The methodological quality of the included SRs was assessed using A Measurement Tool to Assess Systematic Reviews (AMSTAR-2). Data were extracted according to a defined population-intervention-comparison-outcome (PICO) framework from primary studies that included SRs. The same PICO was categorized into PICOs according to interventions (stimulation target, frequency, number of sessions (short: 1-5 sessions, medium: 5-10 sessions, and long: >10 sessions)) and comparison (sham rTMS or other targets). The evidence mapping was presented in tables and a bubble plot. Results. A total of 23 SRs were included. According to the AMSTAR-2, 20 SRs scored "very low" in terms of methodological quality, 2 SRs scored "low," and 1 SR scored "high." A total of 17 PICOs were extracted. The dorsolateral prefrontal cortex (DLPFC) is the most studied of the non-motor cortex targets. PICOs of DLPFC, premotor cortex (PMC), frontal cortex, and secondary somatosensory cortex (S2) were mainly categorized with a "potentially better" conclusion. High-frequency (5-20 Hz) rTMS of non-M1 usually lead to "potentially better" conclusions. Conclusions. DLPFC, PMC, frontal cortex, and S2 seem to be promising new targets for rTMS treatment of certain NP. Evidence mapping is a useful and reliable methodology to identify and present the existing evidence gap that more research efforts are necessary in order to highlight the optimal stimulation protocols for non-M1 targets and standardize parameters to fill the evidence gaps of rTMS. Further investigation is advised to improve the methodological quality and the reporting process of SRs.

\section{Introduction}

Neuropathic pain (NP) is a chronic pain caused by lesions or dysfunction of the peripheral or central nervous system; it is often characterized by persistent pain, hyperalgesia, or even spontaneous pain [1]. NP not only disturbs daily activities, work, and sleep but also increases the incidence of emotional disorders such as patient depression and anxiety [2]. The mechanisms of NP are still unclear, which lead to the challenge of NP prevention and management. Pathological 
changes such as spontaneous activity in damaged non-nociceptive fibers, peripheral and central, hyperactivity in nociceptors, and changes in central neuroplastic may be the possible reasons for NP [3, 4]. Currently, pharmacological treatment is the primary treatment for NP, including tricyclic anti-depressants, anti-convulsants, anti-epileptics, non-steroidal anti-inflammatory drugs, opioids, and so on. $[5,6]$. However, pharmacological treatments provide less satisfaction with pain relief in many patients. In addition, drugs cause many adverse effects and even lead to drug dependence and abuse [7], wherein recommendation levels are not high $[2,8]$. Thus, the treatment of NP remains a major unmet need, and the exploration of alternative approaches, especially evidence-based non-pharmacological interventions, is particularly important.

Repetitive transcranial magnetic stimulation (rTMS), as a non-invasive, safe non-pharmacological treatment, has been widely applied for NP. The rTMS technique uses magnetic pulses from an external stimulator to target specific cortical areas to generate induced currents that can alter the action potential of cortical nerve cells, induce depolarization of neurons, and ultimately lead to functional and even structural plasticity changes in the nervous system [9]. rTMS for NP has been published extensively. Stimulation target, frequency, and session are considered to be critical variables for analgesic efficacy. In terms of target, primary motor cortex (M1) is a commonly used stimulation target for rTMS and has been used for pain relief related to poststroke central pain, postherpetic neuralgia, and trigeminal facial pain. Although M1 has shown some efficacy in the treatment of NP, some studies have found that patients do not respond to M1 stimulation or only have short-lived effects. One study found the overall effectiveness rate was only about $40 \%$ [10]. This leaves a large gap in the search to find better management options for nonresponders. Given the complexity of the disease type and the unclear mechanism of NP, M1 is not the suitable stimulation target for all types of NP. Therefore, the lack of individualized targeted therapy based on the characteristics of plasticity [11] may explain why rTMS is effective only in some patients, with a non-persistent efficacy and pain recurrence.

However, evidence-based evidence on non-M1 stimulation targets, treatment parameters, and treatment efficacy for the treatment of NP is still to be provided. Traditionally, SRs are a common methodology for evidence synthesis. SRs tend to focus on specific types of pain, whereas compared with M1, studies in non-M1 targets are insufficient and unfocused on specific NP, deeming SRs unable to provide a comprehensive overview of non-M1 regions for the treatment of NP. To overcome this barrier, an emerging synthesis method, evidence mapping [12-14], has been developed to provide an overview of the research area. Evidence mapping can provide both the breadth of evidence by extracting and analyzing primary data in SRs and the credibility of evidence by AMSTAR-2. The knowledge gaps identified by using evidence mapping can also inform future studies. This study aims to summarize, identify, and analyze the currently available evidence in SRs regarding rTMS on non-M1 for NP. This information is provided in a user-friendly manner that helps identify research gaps and assist evidence users in decision-making.

\section{Methods}

2.1. Setting the Boundaries and Context of the Evidence Mapping. This evidence mapping is based on the methodology proposed by GEM [15] and previous key studies [16-18]. The study process was divided into five stages (Figure 1). Studies and guidelines related to NP were referred, and an expert with a research background in NP was consulted to frame the evidence mapping. With the help of experts in this area, the specific terminology of the search strategy was confirmed, and the possible evidence users (pain, neurology, psychiatry, anesthesiology, and rehabilitation) involved were discussed. On the basis of the above information, the eligibility criteria have been established for inclusion in the study. Studies containing rTMS for NP were considered eligible. Studies on patients with NP were included, whereas experimental subjects that were animals or healthy people were excluded. The intervention should be rTMS, and the comparison could be rTMS, sham rTMS, other treatments of relieving pain, or no treatment. The outcome should be pain measured with various clinically validated tools such as visual analog scale (VAS), numerical rating scale (NRS), short-form McGill Pain Questionnaire, and brief pain inventory. Studies that did not address intervention outcomes, such as those aimed to explore NPrelated pathophysiology and focusing on cost-effectiveness, were excluded. Studies that reported other outcomes (e.g., fatigue, motor function, spasticity, sensory function, and cognition) with the exception of pain were also excluded. Only SRs (with or without meta-analysis) were included as they could provide more reliable evidence. Literature published in non-English languages were excluded. Posters and conference abstracts were excluded.

2.2. Search and Select Evidence. We conducted searches of systematic literature published before January 23, 2021, on PubMed, EMBASE, Epistemonikos, and the Cochrane Library. Medical subject headings were used in combination with free-text terms for the search, such as "neuralgia," "neurodynia," "atypical neuralgia," "nerve pain," and "stump neuralgia." Literature published in non-English languages were excluded. In addition, references of included studies were also searched to ensure the integrity of the search. The details of the search strategies are provided in Supplementary Material 1.

EndNote (version X9) was used to manage all retrieved results. After removing duplicated SRs, two reviewers (Zang and Lai) independently screened titles and abstracts to exclude irrelevant studies. Full-text studies were obtained and reviewed to make a terminal decision. Any disagreements in the decision-making process were resolved by negotiation or discussion with a third reviewer (Zhang).

2.3. Assessing the Methodological Quality of SRs. The methodological quality for each SRs was assessed with the AMSTAR-2 [19]. Almost half of published SRs have included both randomized controlled trials (RCT) and nonrandomized studies. AMSTAR-2 is suitable for evaluating 


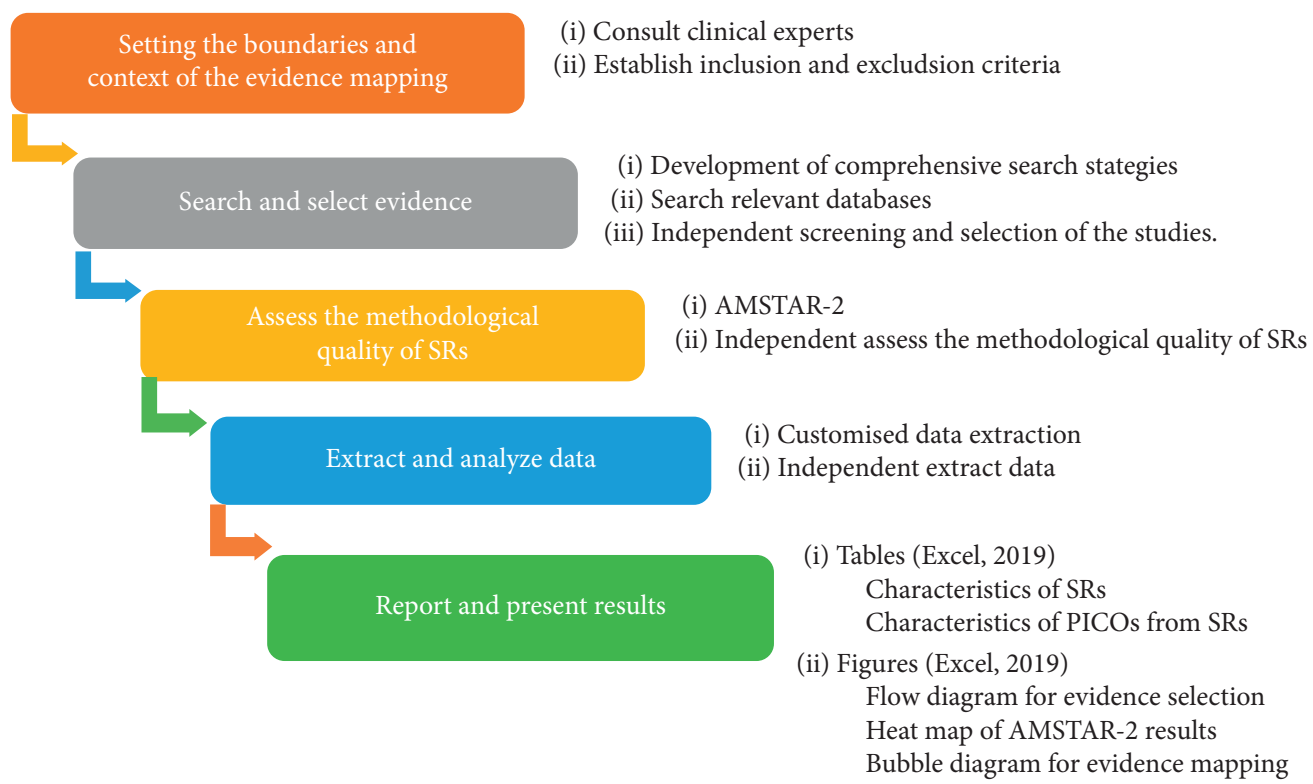

FIgURE 1: Core tasks for conducting evidence mapping.

the methodological quality of SRs that include RCT and nonRCT. A total of 16 items were included, covering the entire process of SRs and including topic selection, design, registration, data extraction, data statistical analysis, and discussion. AMSTAR-2 recommends 7 items (items: 2, 4, 7, 9, 11,13 , and 15) as key items for evaluating the quality of SRs (Figure 2). According to the absence of items, the evaluation results of the SRs are divided into the following four categories: "high," no key items missing and on more than one non-critical item missing; "moderate," no key items missing and more than one non-key item missing; "low," one key item missing and with or without non-key items missing; and "critically low," more than one key item missing and with or without non-critical items missing.

2.4. Extract and Analyze Data. Two data extraction tables were designed to record the main characteristics of the included SRs. Data were grouped into two categories:

(a) General characteristics of the SRs: authors, years of publication, types of SRs (with or without metaanalysis), objectives, dates of search, sample sizes, designs, and numbers of included primary studies.

(b) Characteristics of research questions: the PICO framework was used to extract data from primary studies that had been included in SRs. The four key components are study population, interventions, comparative measures, and assessment methods for outcomes. Due to the unavoidable heterogeneity of rTMS protocol among studies, it is difficult to classify and categorize all parameters. Targets, frequency, and sessions were most often reported for each primary study included in the SRs. They have been shown to influence analgesic effects and are identified as the most clinically significant factors [20-23]. High and low frequencies of rTMS could induce transient excitatory and inhibitory effects, respectively [24]. Sessions of rTMS are considered to be an important factor in maintaining the effects. Thus, the PICO characteristics are mainly focused on interventions (targets, frequency, and sessions) and comparison (sham rTMS or other targets).

According to the criteria reported previously, the conclusions of rTMS on NP reported by the systematic evaluation were classified into five categories: "potentially better," "mixed results," "unclear," "no difference," and "potentially worse." "Potentially better" is defined as statistically significant efficacy of rTMS, with the authors of the SR having no doubt about the current evidence and recommending the therapy. "Mixed results" means that the results of SRs with similar content are controversial (e.g., some SRs found no difference between transcranial magnetic stimulation with the control group in the same study, whereas others found potential benefits of transcranial magnetic stimulation over the control group. "Unclear" is defined as the SR authors concluding that the evidence is inconclusive or that the conclusions of a specific study were not reported by the authors of the SR. "No difference" is defined as comparable efficacy of rTMS as compared to the control group or no statistical difference. "Potentially worse" is defined as better efficacy in the control group as compared with rTMS. When SRs yielded consistent results for the same study, it was added to the appropriate group, and conflicting results were included in the "mixed results" group.

Two authors (Zang and Lai) assessed the methodological quality and extracted data independently. Any difference of 


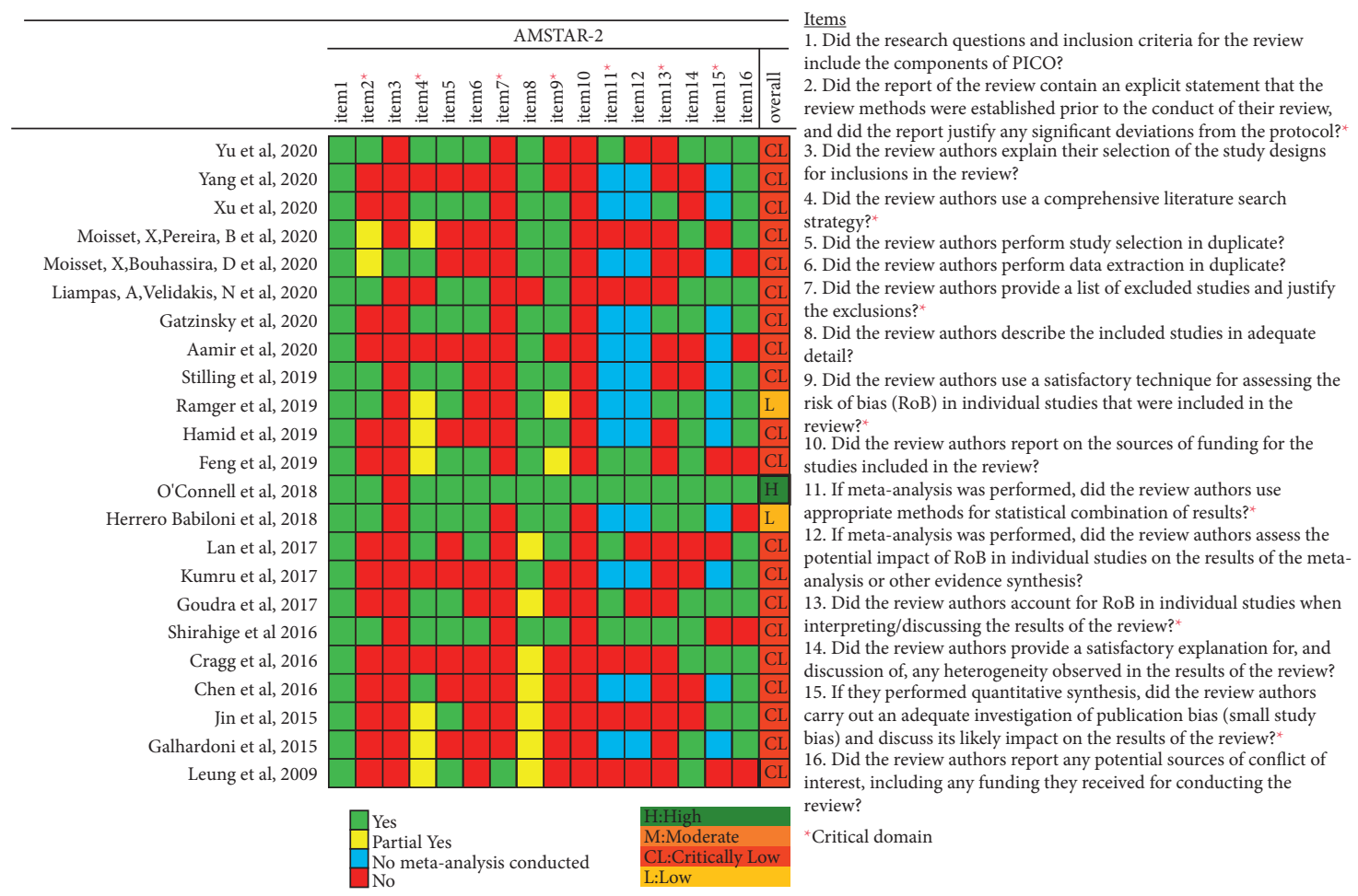

FIgURE 2: Methodological quality of the included systematic reviews.

opinions was discussed with the third author (Zhang). The original authors were contacted for missing information when necessary.

2.5. Report and Present Results. The evidence mapping was presented in three visualizations, and the findings were summarized in a narrative synthesis:

(a) Tables were used to describe the basic characteristics of the included SRs and characteristics of all identified PICOs.

(b) A heat map was displayed to present the quality of SRs.

(c) A bubble plot was used to present a comprehensive visualization of the conclusions of included SRs, methodological quality, sample size, and distribution of interventions. The bubble plot can display the following information: (1) authors' conclusions: ratings on the $x$-axis are: "potentially better," "mixed results," "unclear," "no difference," or "potentially worse"; (2) AMSTAR-2 evaluation results: presented in four different colors on the $y$ axis (red indicating critically very low, orange indicating low, yellow indicating medium, and green indicating high quality); (3) research characteristics: different colored bubbles indicate different PICOs; (4) the number of primary studies included in the SRs, shown in each bubble and indicated by the bubble size; and (5) interpretation of bubble plot: some primary studies may be included in multiple SRs. If SRs synthesized different conclusions for the same primary study, the same PICOs would appear in different classifications on the $X$-axis. If the same primary study was included by SRs of different quality, then the same PICOs classified by the primary study would appear in different classifications on the $Y$-axis. The included SRs covering similar topics may have overlapped considerably in terms of the primary studies they contained. Therefore, when interpreting the evidence mapping, it is critical that all figures in the bubble are not added up and that any overlapping studies are removed. Due to SRs serving as the unit of analysis rather than the primary study, the risk of bias is reduced when multiple reviews reach the same conclusion. When higher-quality SRs cover the same primary study, these findings may be interpreted with more confidence than the findings of lower quality reviews [25]. Conversely, the potential for bias appears when the primary study is concluded by only one low-quality SR, presented as "critically low" at the bottom of the bubble plot. Multiple bubbles with different results may indicate that this type of evidence highlights the preliminary stage or unclear nature of the evidence.

\section{Results}

3.1. Studies Selected. The study selection process is shown in Figure 3. The list of excluded studies and the reasons for exclusion are provided in Supplementary Material 2. 


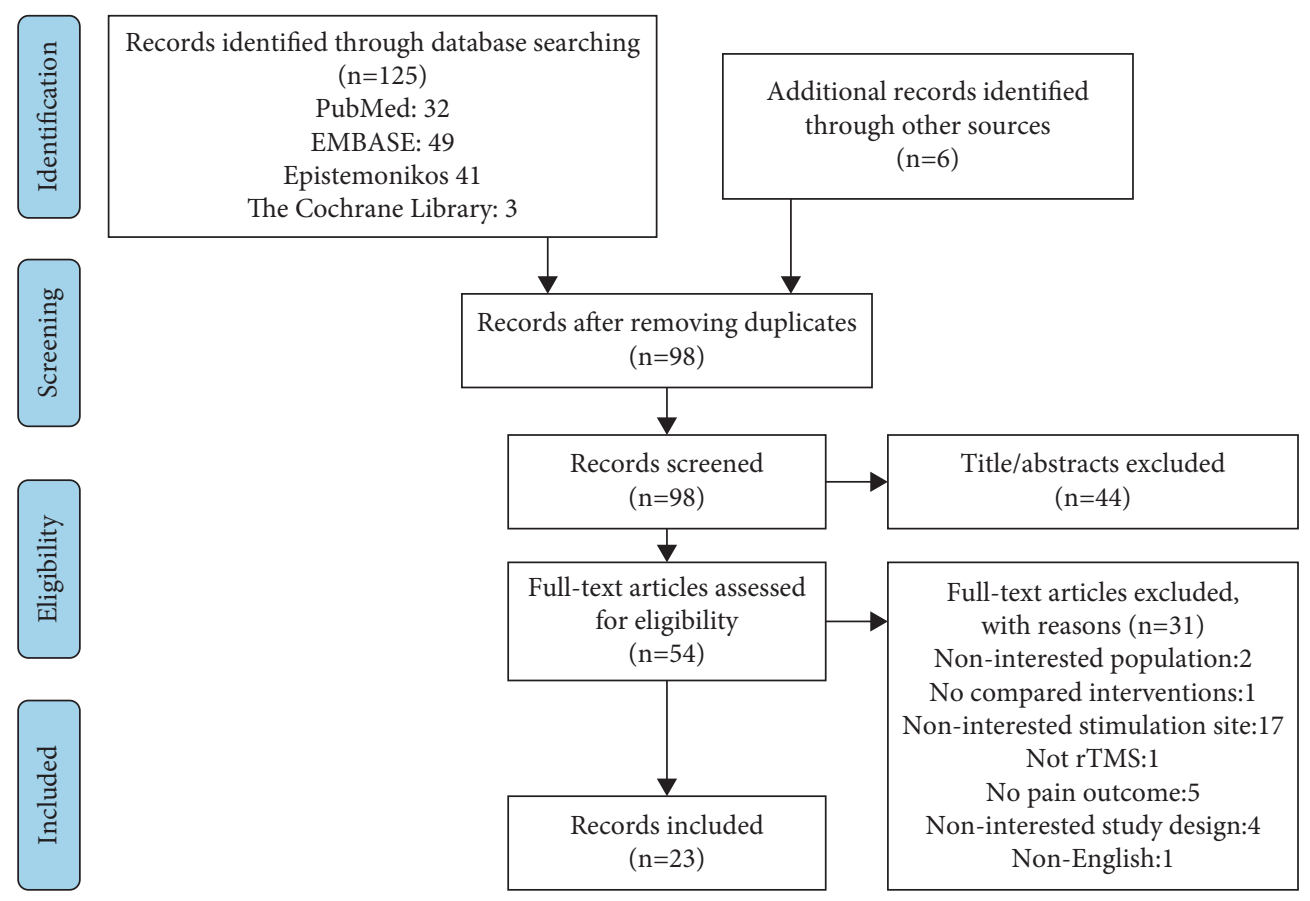

FIGURE 3: Flow diagram for evidence selection.

3.2. The Methodological Quality of SRs. As shown in Figure 2, according to AMSTAR-2 criteria, 1 Cochrane SR [23] was graded as "high." Two SRs $[26,27]$ were graded as "low," and 20 SRs [18, 27-45] were graded as "critically low" $[22,28-46]$. The SRs were downgraded mainly due to the following reasons: absence of a predesigned and registered protocol [22, 29, 30, 34, 36, 40, 42-46]; no explanation for the selection of study design included in the SRs $[22,23,26,31,33-46]$; no list of the excluded studies or reasons for the exclusion [22, 26-45]; no statement of the funding or support for each included primary study in the SRs [22, 26-46]; and no investigation of the impact of the risk of bias in the included studies on the overall effect $[28,29,31,40,42-46]$. The detailed evaluation process is provided in Supplementary Material 3.

3.3. Characteristics of SRs. Table 1 shows the characteristics of the included SRs. All SRs [22, 23, 26-46] were published between 2009 and 2020. Among the 23 included SRs, 11 $[23,28,31,33,37,38,40,42,44,46]$ conducted a metaanalysis. The number of included primary studies ranged from 5 to 131, and they were conducted between 2004 and 2020. Each SR included patients ranging from 109 to 15,776 . A total of 3 SRs $[33,39,43]$ did not report or incompletely reported the designs of the included studies. Among the available data, a total of 509 randomized controlled trials (RCTs) accounted for $80.3 \%$ of the included studies in all SRs. Of all SRs, 13 [23, 27, 28, 30, 32, 36, 38, 41, 42, 45, 46] included only RCTs; 12 SRs [22, 23, 29, 31, 34, 36, 39, 40, 42, 44-46] included patients with NP with different causes; and 11 were specially conducted on NP with specific etiologies or due to a single disease. One SR [28] included pain after spinal cord injury (SCI); 4 SRs [26, 30, 33, 43] included central poststroke pain after stroke (CPSP); 4 SRs [31, 37, 38, 41] included migraine, 1 SR [35] included headache, and 1 SR [27] included orofacial pain (OFP). As for the intervention, 9 SRs [22, 29, 34, 36, 38, 40, 44-46] only assessed TMS; 8 SRs $[23,26,28,35,37,39,41]$ also assessed other non-invasive stimulations; 1 SR [31] assessed neuromodulation techniques; 1 SR [30] assessed non-pharmacological interventions; 3 SRs $[32,33,42]$ assessed pharmacological and non-pharmacological management of NP; and 1 SR [43] assessed non-invasive physical modalities.

3.4. Characteristics of PICOs from SRs. After merging the duplicated primary studies included in the 23 SRs, 24 primary studies that provide the mandatory parameter information were integrated into 17 PICOs groups according to the PICO characteristics.

The key characteristics of PICOs are listed in Table 2. The details of the characteristics are enumerated in Supplementary Material 4. In terms of the stimulation target, 6 PICOs stimulated the left DLPFC; 2 PICOs stimulated the S2; 2 PICOs stimulated the vertex; 1 PICO stimulated the PFC; 1 PICO stimulated the frontal cortex; 4 PICOs stimulated multiple different targets; and 1 PICO stimulated over the superior trapezius muscle. In terms of the stimulation frequency, 14 PICOs used high-frequency rTMS $(>1 \mathrm{~Hz}) ; 2$ PICOs used low-frequency rTMS $(<1 \mathrm{~Hz})$; and 1 PICO used both high and low frequencies. In terms of the number of sessions, 1-5 sessions were considered as short sessions, 6-10 as medium sessions, and more than 10 as long sessions. Three PICOs had long sessions; 6 PICOs had medium sessions; and 8 had short sessions. All PICOs used sham stimulation or placebo as a control to study the effectiveness of rTMS in patients with NP. In addition, 3 PICOs also 
TABLE 1: Characteristics of included systematic reviews.

\begin{tabular}{|c|c|c|c|c|c|c|}
\hline Author and year & $\begin{array}{l}\text { Study } \\
\text { design }\end{array}$ & Search date & Objective & $\begin{array}{l}\text { Number of } \\
\text { studies } \\
\text { included }\end{array}$ & $\begin{array}{l}\text { Design and number of } \\
\text { included studies }\end{array}$ & $\begin{array}{l}\text { Participants } \\
(n)\end{array}$ \\
\hline $\begin{array}{l}\text { Yu et al., } 2020 \\
\text { [28] }\end{array}$ & SRM & January 2019 & $\begin{array}{l}\text { To investigate the effect of non- } \\
\text { invasive brain stimulation for SCI }\end{array}$ & 11 & RCT: 11 & 274 \\
\hline $\begin{array}{l}\text { Yang et al., } 2020 \\
\text { [29] }\end{array}$ & SR & June 2019 & $\begin{array}{c}\text { To explore the effect of rTMS on } \\
\text { different types of pain }\end{array}$ & 106 & $\begin{array}{c}\text { RCT: 69; OLT: 16; CR: } \\
21\end{array}$ & 3,264 \\
\hline $\begin{array}{l}\text { Xu et al., } 2020 \\
{[30]}\end{array}$ & SR & August 2020 & $\begin{array}{l}\text { To assess the efficacy and safety of } \\
\text { non-pharmacological therapies for } \\
\text { CPSP }\end{array}$ & 11 & RCT: 11 & 210 \\
\hline $\begin{array}{l}\text { Moisset et al., } \\
2020 \text { [31] }\end{array}$ & SRM & July 2020 & $\begin{array}{c}\text { To investigate the efficacy of } \\
\text { neurostimulation techniques in } \\
\text { migraine }\end{array}$ & 38 & RCT: 38 & 2,899 \\
\hline $\begin{array}{l}\text { Moisset et al., } \\
2020[32]\end{array}$ & SR & August 2019 & $\begin{array}{l}\text { To propose all the alternative } \\
\text { treatment options for NP }\end{array}$ & 131 & RCT: 131 & 15,776 \\
\hline $\begin{array}{l}\text { Liampas et al., } \\
2020[33]\end{array}$ & SRM & November 2019 & $\begin{array}{l}\text { To describe the prevalence and } \\
\text { characteristics of CPSP and } \\
\text { investigate the relevant } \\
\text { management methods }\end{array}$ & 69 & NR & NA \\
\hline $\begin{array}{l}\text { Gatzinsky et al., } \\
2020[22]\end{array}$ & SR & June 2019 & $\begin{array}{c}\text { To review the efficacy and safety of } \\
\text { rTMS on M1 }\end{array}$ & 32 & RCT: 24; CS: 8 & $682(\mathrm{RCT})$ \\
\hline $\begin{array}{l}\text { Aamir et al., } \\
2020[34]\end{array}$ & SR & June 2019 & $\begin{array}{c}\text { To evaluate the effect of rTMSfor } \\
\text { peripheral NP }\end{array}$ & 12 & RCT: 5; CS: 2; CR: 5 & 188 \\
\hline $\begin{array}{l}\text { Stilling et al., } \\
2020[35]\end{array}$ & SR & September 2018 & $\begin{array}{l}\text { To review the use of TMS and tDCS } \\
\text { for specific headache disorders }\end{array}$ & 34 & $\begin{array}{l}\text { Randomized trials: } 20 \\
\text { NRC/prospective } \\
\text { cohort/OLT: } 14\end{array}$ & 1,787 \\
\hline $\begin{array}{l}\text { Ramger et al., } \\
2019 \text { [26] }\end{array}$ & SR & 2018 & $\begin{array}{l}\text { To evaluate the efficacy of rTMS and } \\
\text { tDCS for CPSP }\end{array}$ & 6 & $\begin{array}{l}\text { RCT: } 1 \text {; prospective } \\
\text { cohort: } 1 \text {; CS: } 2 \text {; cross- } \\
\text { over: } 2\end{array}$ & 109 \\
\hline $\begin{array}{l}\text { Hamid et al., } \\
2019[36]\end{array}$ & SR & 2018 & $\begin{array}{l}\text { To explore the effect of rTMS on } \\
\text { chronic refractory pain, especially } \\
\text { in adults with central NP }\end{array}$ & 12 & RCT: 12 & 350 \\
\hline $\begin{array}{l}\text { Feng et al., } 2019 \\
\text { [37] }\end{array}$ & SRM & September 2018 & $\begin{array}{c}\text { To evaluate the efficacy of rTMS and } \\
\text { tDCS for migraine }\end{array}$ & 9 & RCT: 9 & 276 \\
\hline $\begin{array}{l}\text { O'Connell et al., } \\
2018 \text { [23] }\end{array}$ & SRM & October 2017 & $\begin{array}{l}\text { To assess the efficacy of non- } \\
\text { invasive cortical stimulation } \\
\text { techniques on chronic pain }\end{array}$ & 94 & RCT: 94 & 2,983 \\
\hline $\begin{array}{l}\text { Herrero } \\
\text { babiloni et al., } \\
2018[27]\end{array}$ & SR & NR & $\begin{array}{l}\text { To explore the effect of TMS and } \\
\text { tDCS for chronic OFP }\end{array}$ & 14 & RCT: 14 & 228 \\
\hline $\begin{array}{l}\text { Lan et al., } 2017 \\
\text { [38] }\end{array}$ & SRM & April 2017 & $\begin{array}{l}\text { To explore the efficacy of TMS for } \\
\text { migraine }\end{array}$ & 5 & RCT: 5 & 313 \\
\hline $\begin{array}{l}\text { Kumru et al., } \\
2017 \text { [39] }\end{array}$ & SR & August 2015 & $\begin{array}{c}\text { To assess the role of rTMS or } \\
\text { peripheral magnetic stimulation for } \\
\text { NP }\end{array}$ & 39 & NR & 892 \\
\hline $\begin{array}{l}\text { Goudra et al., } \\
2017[40]\end{array}$ & SRM & NR & $\begin{array}{l}\text { To evaluate the effect of rTMS for } \\
\text { chronic pain }\end{array}$ & 9 & $\begin{array}{l}\text { RCT: 6; prospective } \\
\text { observational: } 3\end{array}$ & 183 \\
\hline $\begin{array}{l}\text { Shirahige et al., } \\
2016\end{array}$ & SRM & $\begin{array}{l}\text { November } 11 \text {, } \\
\text { 2015, to January } \\
15,2016\end{array}$ & $\begin{array}{c}\text { To assess the effect of NIBS on } \\
\text { migraine patients }\end{array}$ & 8 & RCT: 8 & 296 \\
\hline $\begin{array}{l}\text { Cragg et al., } \\
2016[42]\end{array}$ & SRM & May 2015 & $\begin{array}{c}\text { To explore the predictors of placebo } \\
\text { responses in central NP clinical } \\
\text { trials }\end{array}$ & 39 & RCT: 39 & 1,153 \\
\hline $\begin{array}{l}\text { Chen et al., } 2016 \\
{[43]}\end{array}$ & SR & September 2015 & $\begin{array}{c}\text { To evaluate the antalgic effects of } \\
\text { non-invasive physical modalities on } \\
\text { CPSP }\end{array}$ & 16 & NA & 184 \\
\hline $\begin{array}{l}\text { Jin et al., } 2015 \\
\text { [44] }\end{array}$ & SRM & December 2014 & $\begin{array}{c}\text { To evaluate the optimal parameters } \\
\text { of rTMS for NP }\end{array}$ & 25 & $\begin{array}{l}\text { RCT: } 20 \text {; self- } \\
\text { controlled: } 5\end{array}$ & 589 \\
\hline $\begin{array}{l}\text { Galhardoni } \\
\text { et al., } 2015 \text { [45] }\end{array}$ & SR & 2014 & $\begin{array}{c}\text { To review the studies on the } \\
\text { analgesic effects of rTMS in chronic } \\
\text { pain }\end{array}$ & 33 & RCT: 33 & 842 \\
\hline
\end{tabular}


TABLE 1: Continued.

\begin{tabular}{|c|c|c|c|c|c|c|}
\hline Author and year & $\begin{array}{l}\text { Study } \\
\text { design }\end{array}$ & Search date & Objective & $\begin{array}{l}\text { Number of } \\
\text { studies } \\
\text { included }\end{array}$ & $\begin{array}{l}\text { Design and number of } \\
\text { included studies }\end{array}$ & $\begin{array}{l}\text { Participants } \\
(n)\end{array}$ \\
\hline $\begin{array}{l}\text { Leung et al., } \\
2009[46]\end{array}$ & SRM & August 2007 & $\begin{array}{l}\text { To evaluate the overall effect of } \\
\text { rTMS for NP and evaluate the effect } \\
\text { of treatment parameters. }\end{array}$ & 5 & RCT: 5 & 149 \\
\hline
\end{tabular}

SRs: systematic reviews (with or without meta-analysis); SR: systematic review; SRM: systematic review with meta-analysis; NP: neuropathic pain; SCI: spinal cord injury; rTMS: repetitive transcranial magnetic stimulation; CPSP: central poststroke pain; M1: motor cortex; TMS: transcranial magnetic stimulation; tDCS: transcranial direct current stimulation; OFP: orofacial pain; NIBS: non-invasive brain stimulation; RCT: randomized controlled trial; OLT: open-label trial; CR: case report; NR: not reported; CS: case series; and NA: not available.

compared the efficacy of different targets. The PICOs were concentrated in the following characteristics: $10 \mathrm{~Hz}$, shortterm sessions (4 PICOs from 7 primary studies); $10 \mathrm{~Hz}$, medium-term sessions (3 PICOs from 6 primary studies); and $10 \mathrm{~Hz}$, long-term sessions (2 PICOs from 5 primary studies).

\subsection{Specific Findings from SRs in the Evidence Mapping.} The evidence mapping of the rTMS for NP is presented in Figure 5. The bubble diagram is a visual display of data represented in Table 2. Evidence mapping showed that DLPFC is the most studied of the targets (5 PICOs from 9 primary studies) and showing a majority of "potentially better" treatment effects. In addition, the PICOs of PMC, frontal cortex, and S2 in trigeminal NP were mainly categorized with a "potentially better" conclusion and seem to be promising new targets for rTMS treatment of certain NP. PFC, S1, SMA, preM, and S2 in chronic visceral pain were categorized as "mixed" conclusions.

High-frequency $(5-20 \mathrm{~Hz})$ rTMS of non-M1 usually lead to "potentially better" conclusions as compared with sham stimulation, although some had transient effects. In contrast, the synthesis results for the lower frequencies $(1 \mathrm{~Hz})$ showed either no difference, unclear, or mixed.

Nine PICOs included 10 primary studies rated as "potentially better," and one of these PICOs involved one primary study that was also included in a high-quality metaanalysis [47]. In accordance with the AMSTAR-2 quality assessment, the interventions in these four PICOs were considered beneficial in most cases. Six PICOs included 7 primary studies with different findings within different SRs that were rated as "mixed," and this implies that the interventions in these eight PICOs had limited confidence in the effect estimates; true effects may be different from the study reports [48]. One PICO conclusion was rated as "unclear" due to its effect and was not reported in the SR [23]. Six PICOs included 7 primary studies that concluded that rTMS showed no difference when compared with the controls. Of these, 4 PICOs included 5 primary studies showing a potentially better effect of rTMS in the short term but no difference during long-term follow-up (Table 2; Supplementary Material 4). After the exclusion of studies that were ineffective during follow-up, no primary studies were included by a high-quality meta-analysis [47]. Two PICOs included 2 primary studies that showed a "potentially worse" conclusion. This finding indicated less effectiveness of these intervention protocols or inapplicability to particular NP, and the treatment effects could be uncertain [49].

\section{Discussion}

As far as we know, this evidence mapping may be the first synthesis of evidence on non-M1 targets for the treatment of NP. Following the classification criteria for interventions, this evidence mapping has described and organized existing evidence for non-M1 targets for NP. The majority of nonM1 targets reported as "potentially better" were DLPFC, probably due to DLPFC can coordinate the interaction between the cognitive pathway and the "pain matrix", or play a direct role in promoting or inhibiting pain through the nociceptive downstream inhibitory pathway [25, 47]. In addition, the PMC, the left frontal cortex, and the S2 also seem to be promising therapeutic targets. These targets are also importantly involved in nociceptive modulation; they share some common mechanisms, such as the involvement in altering human temperature pain thresholds [50], inducing striatal dopamine release that modulates pain [51], and causing cerebral hemodynamic changes in broader cortical regions (e.g., cingulate cortex, anterior frontal cortex, thalamus, and other subcortical areas involved in pain modulation) [52]. Another important finding was that most studies tend to suggest that high-frequencies produce better effects [49, 53-61]. This may be due to the factor that high-frequency rTMS can directly excite the injured hemisphere, thus directly improving pain. However, this evidence mapping does not significantly find the specific number of sessions that may possibly lead to a "potentially better" conclusion. This may be due to the small number of primary studies of different PICOs, making the differences insignificant.

We were able to identify some research gaps by this evidence mapping to orient further research. (1) The included SRs have covered most non-M1 targets of NP, including the DLPFC, ACC, PSI, S2, and S1, while there are still evidence gaps for other targets, such as the premotor cortex (PMC), the supplementary motor complex (SMC), and so on. (2) Low-frequency rTMS inhibited the non-injured side and the high-frequency rTMS excited the injured side. Evidence needs to be evaluated as to whether low and high frequency are used simultaneously to achieve a rebalancing of reciprocal inhibition in both hemispheres and whether the therapeutic effect can be enhanced. (3) Studies have only been conducted from a single cortical target to a 


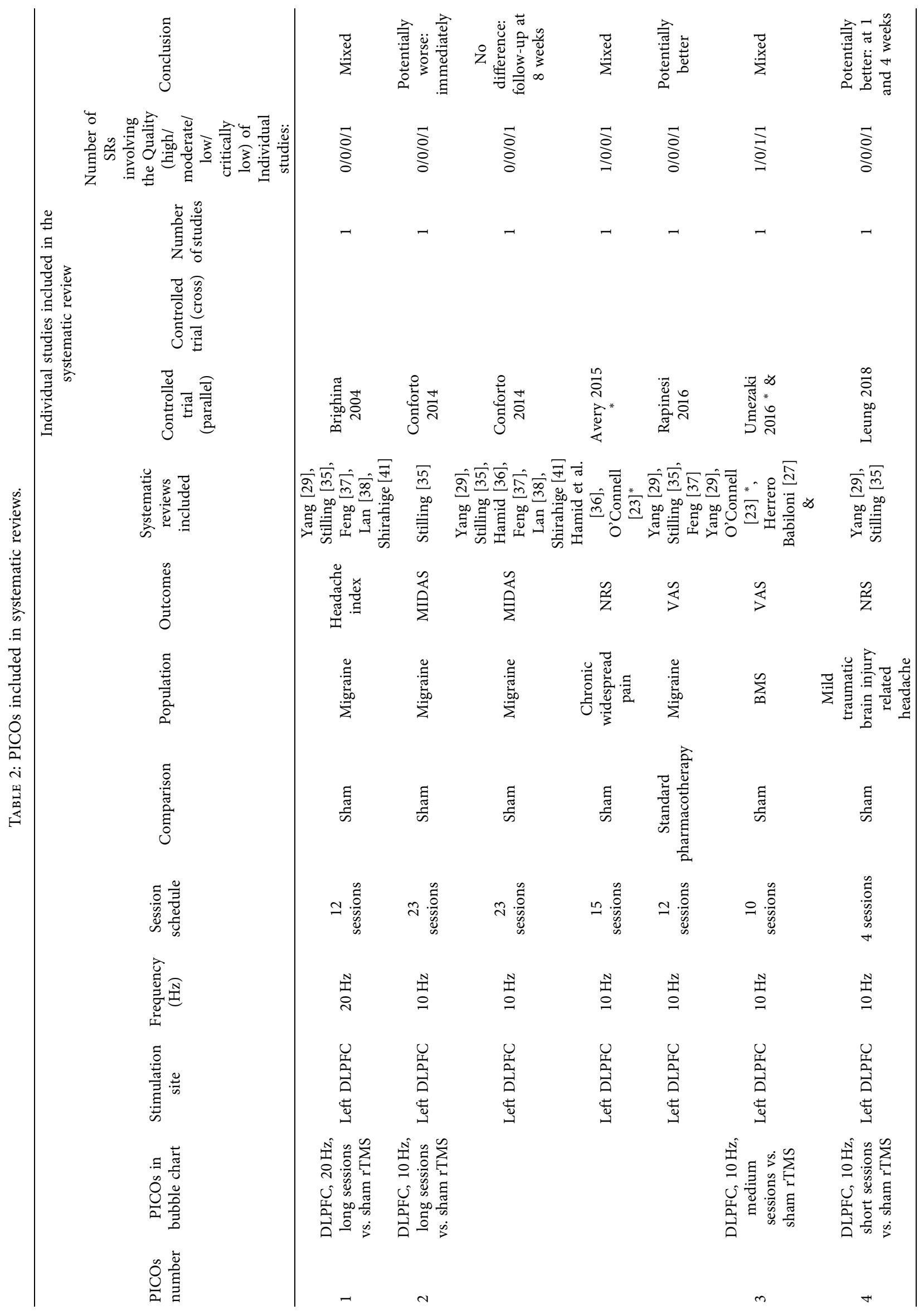




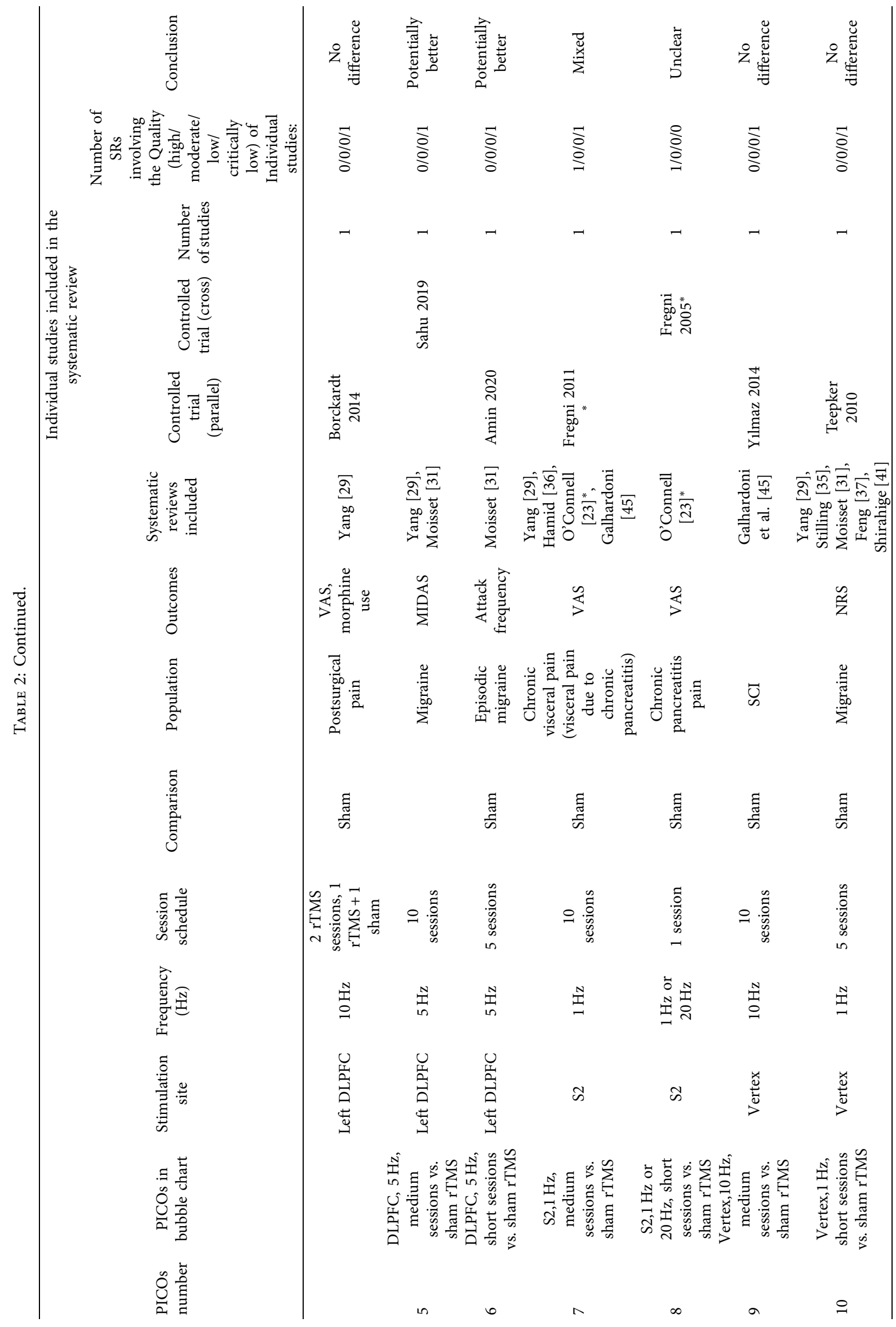




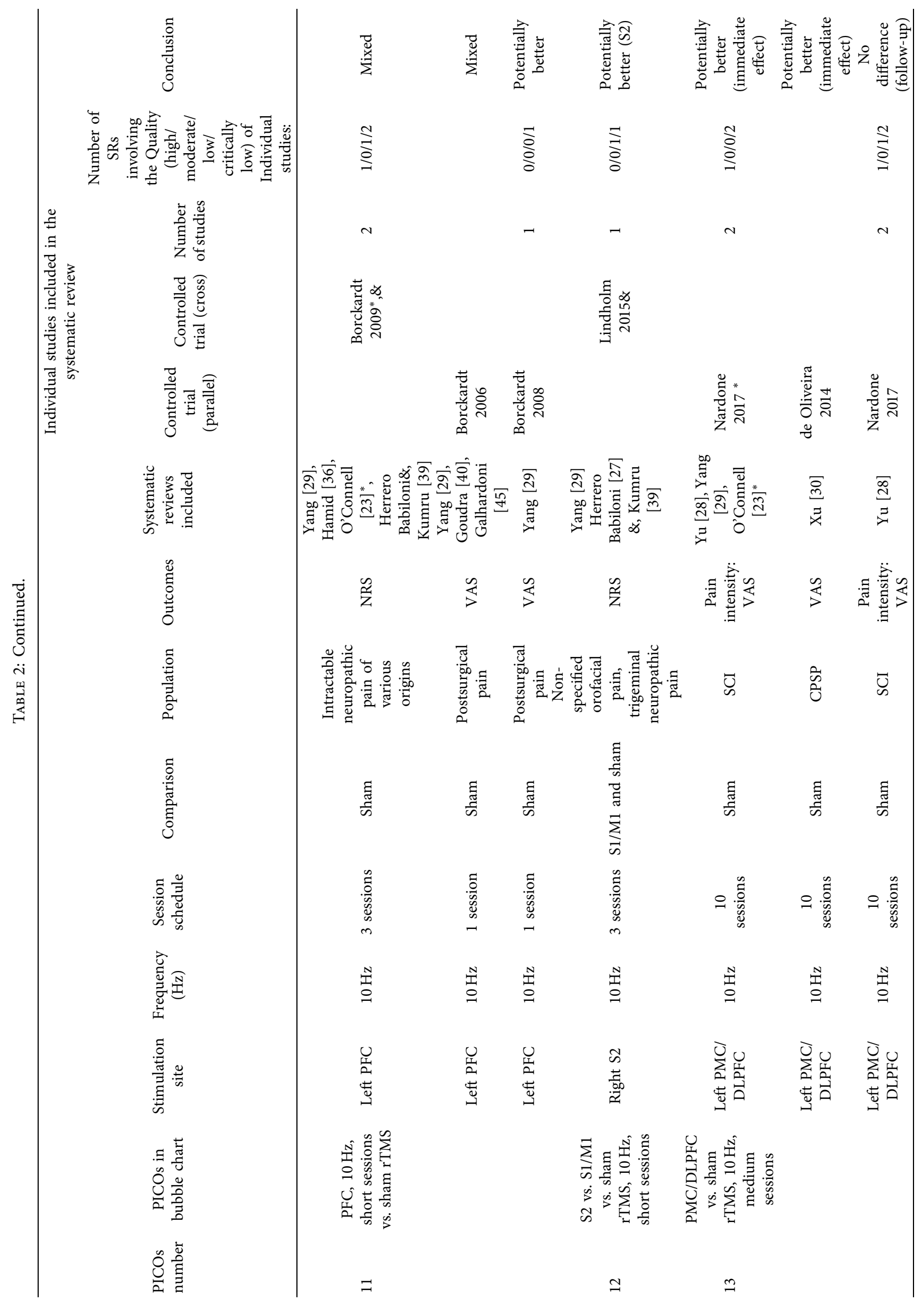




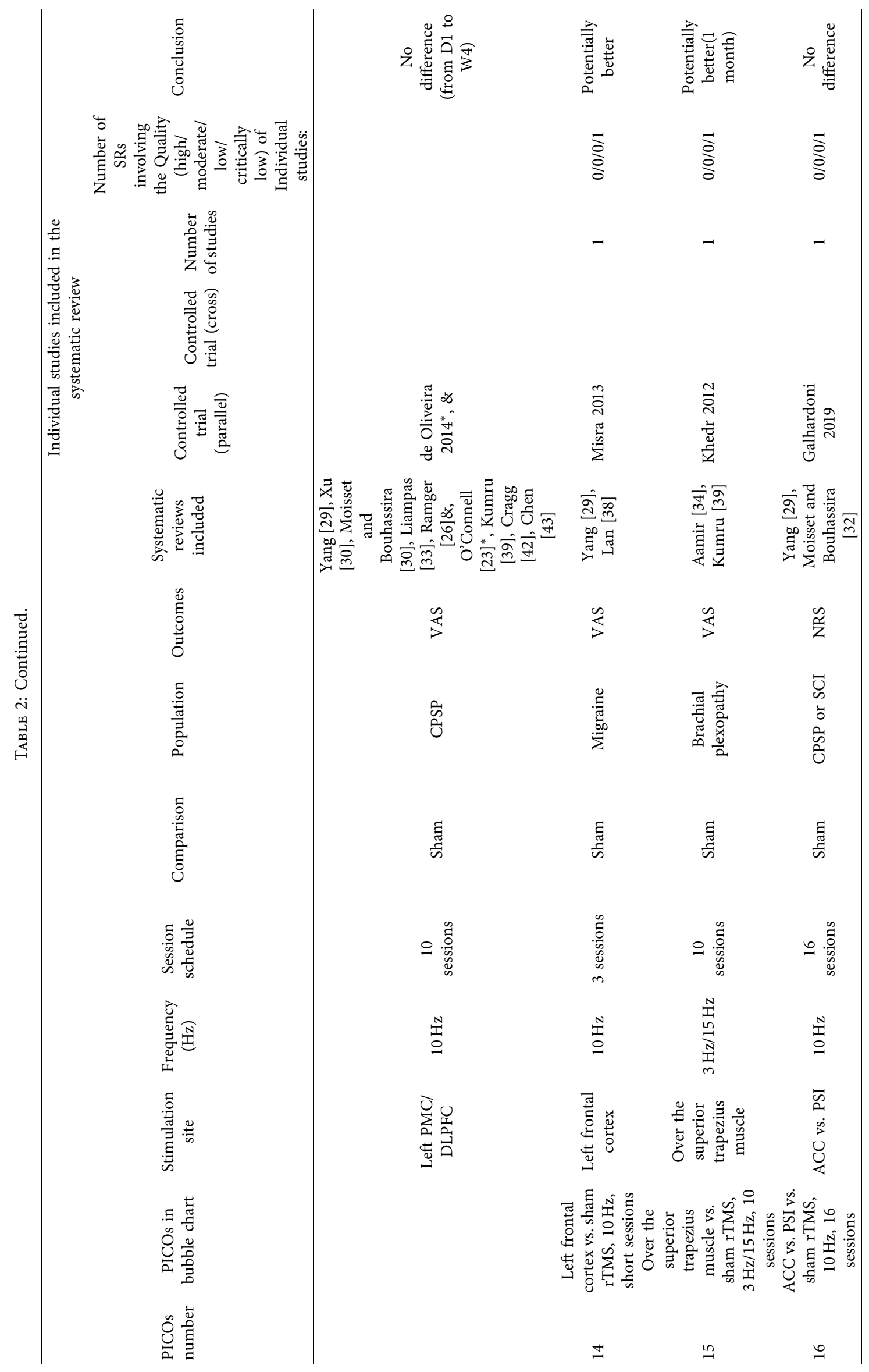




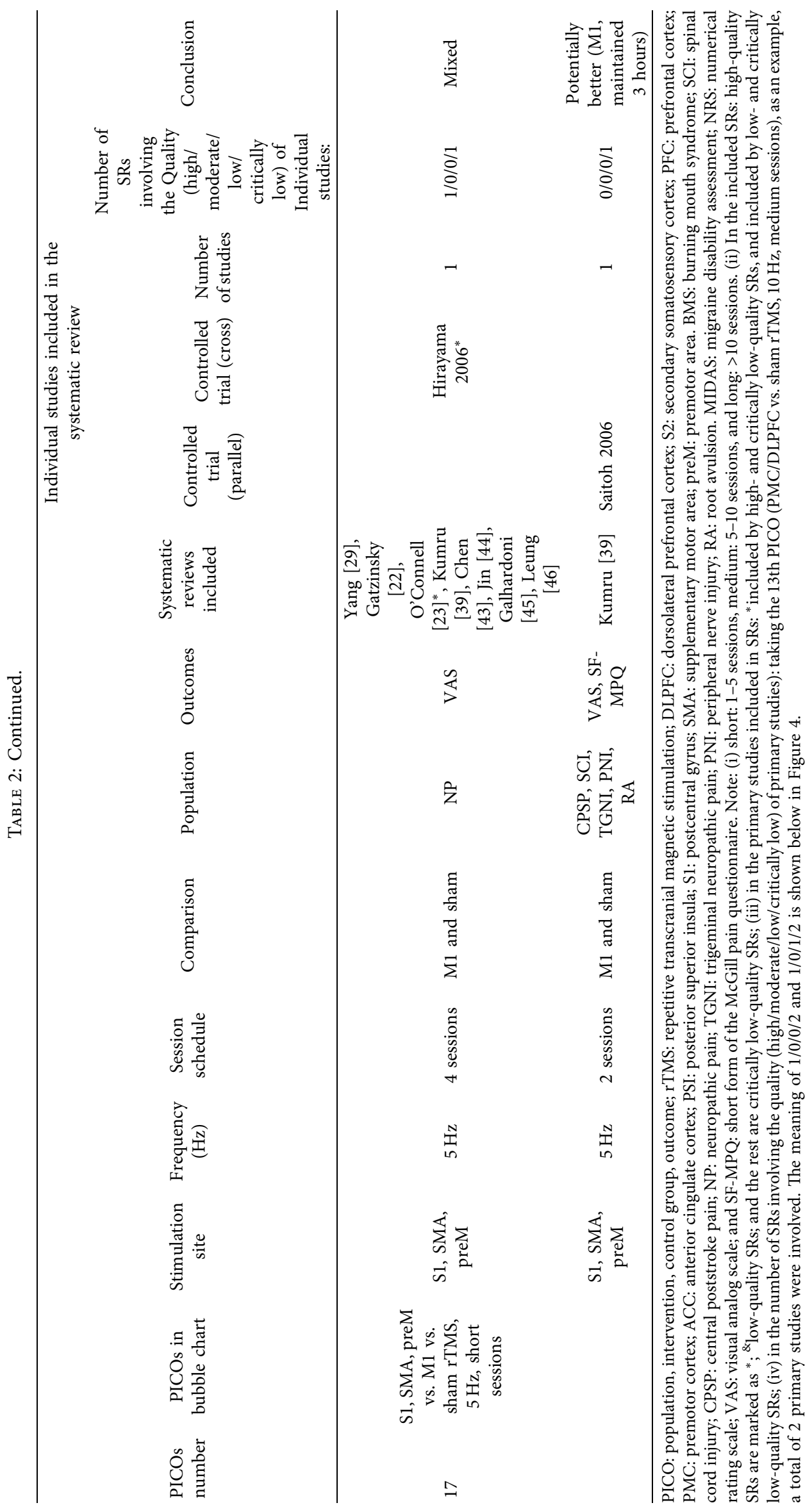



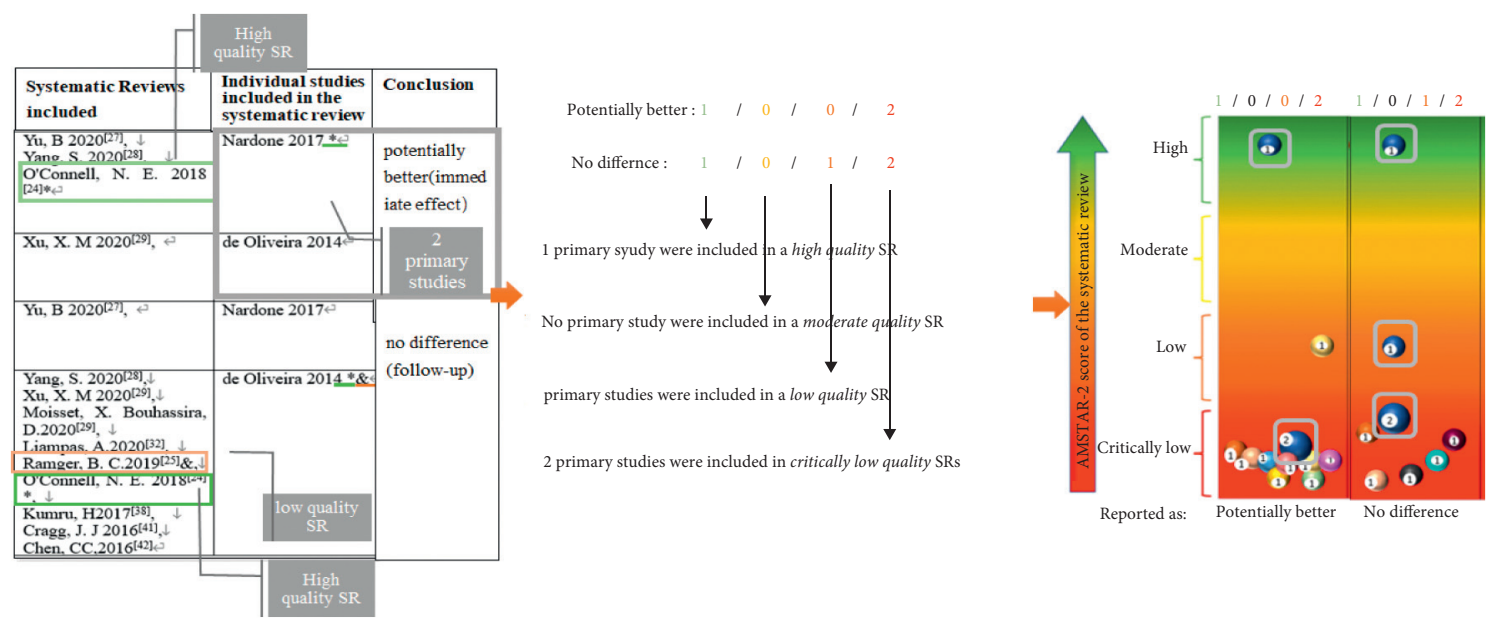

Figure 4: Interpretation of evidence mapping.

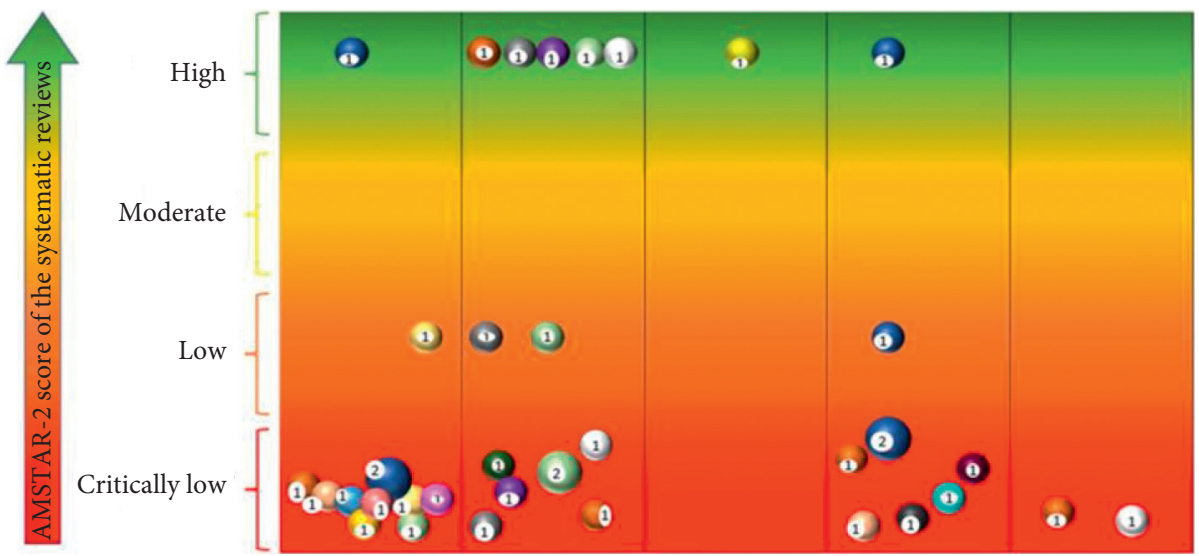

Reported as: Potentially better Mixed Unclear No difference Potentially Worse

- DLPFC,20Hz,long sessions vs sham rTMS O S2 vs S1/M1 vs sham rTMS

- DLPFC, $10 \mathrm{~Hz}$,long sessions vs sham rTMS $10 \mathrm{~Hz}$,short sessions

- PMC/DLPFC vs sham rTMS,

DLPFC,10Hz,medium sessions vs sham rTMS

DLPFC, $10 \mathrm{~Hz}$,short sessions vs sham rTMS

$10 \mathrm{~Hz}$, medium sessions

- Left frontal cortex vs sham rTMS, $10 \mathrm{~Hz}$,short sessions

- DLPFC,5Hz,medium sessions vs sham rTMS

DLPFC, $5 \mathrm{~Hz}$,short sessions vs sham rTMS

- $\mathrm{S} 2,1 \mathrm{~Hz}$,medium sessions vs sham rTMS

O $2,1 \mathrm{~Hz}$ or $20 \mathrm{~Hz}$,short sessions vs sham rTMS

- Vertex,10Hz,medium sessions vs sham rTMS

- Vertex, $1 \mathrm{~Hz}$,short sessions vs sham rTMS

Over the superior trapezius muscle vs sham rTMS, $3 \mathrm{HZ} / 15 \mathrm{HZ}, 10$ sessions

- ACC vs PSI vs sham rTMS,

$10 \mathrm{~Hz}, 16$ sessions

O S1, SMA, preM vs M1 vs sham rTMS,

O PFC, $10 \mathrm{~Hz}$,short sessions vs sham rTMS

FIgURE 5: Evidence mapping of the rTMS on NP. Short: $1-5$ sessions, medium: $5-10$ sessions, and long: $>10$ sessions. $r$ TMS $=$ repetitive transcranial magnetic stimulation; DLPFC: dorsolateral prefrontal cortex; S2: secondary somatosensory cortex; PFC: prefrontal cortex; PMC: premotor cortex; ACC: anterior cingulate cortex; PSI: posterior superior insula; S1: postcentral gyrus; SMA: supplementary motor area; and preM: premotor area.

deep area of the brain; however, the fact is that any deep area is functionally connected to multiple superficial cortices. It would be interesting to examine whether combining different stimulation targets for treatment would enhance the analgesic effect. Similarly, combining transcranial direct current stimulation (tDCS) or prestimulation of a target to enhance the analgesic effect of rTMS could be experimented. (4) In addition to stimulating the target site, special stimulation coils can be designed for the deep brain structures involved in pain information processing, such as anterior cingulate cortex (ACC). (5) Clinical trials of nonM1 targets are mostly small samples with insufficient evidence reliability and an older publication year. Therefore, in future clinical studies, it is necessary to conduct large-scale, multicenter, randomized placebo-controlled trials while establishing safe and effective stimulation parameters, 
selecting homogenous subjects, and reporting the treatment plan with detail and clarity. (6) When multiple SRs in an evidence mapping overlap in the inclusion of primary studies, it may be necessary to cross-check these SRs to determine whether the reported conclusions are the same as well as the extent of the overlap and the impact of the quality of SRs on the applicability of the conclusions. For example, 7 SRs categorized as second PICOs (DLPFC, $10 \mathrm{~Hz}$, long sessions) had different findings, with one in each of the categories of "potentially better," "mixed," "no difference," and "potentially worse." Future reviews could integrate the studies included in all 7 SRs and form new findings. (7) PICOs with multiple bubbles, and particularly those drawing mixed conclusions, may be an area where SRs need to be updated.

The quality of SRs is also an important consideration when conducting SRs. Assessment in this field suggested room for improving SR quality. Future SRs should place more emphasis on the following domains to improve the quality of studies and the validity of the results: reporting explicit statements about the description of the methodology should be designed prior to the conduction of the review; any significant deviations from the protocol should be justified to explain the selection of the study designs for inclusion in the review; a list of excluded studies should be provided and the exclusions should be justified; sources of funding or support for the individual studies included in the SRs should be indicated; and the effect of the risk of bias in individual studies on the total effect should be interpreted and discussed.

4.1. Limitations of the Study. Certain limitations in this evidence mapping should be taken into account. First, our SRs search was done in 2021. However, respective study searches were conducted in 2020 or earlier. Thus, studies that were newly published but may not be included in the SRs may have been overlooked. Nevertheless, we believe that these limitations do not substantially impact our results. Secondly, several different types of studies in SRs comparing therapeutic interventions for NP were included. Although most trials were RCTs, observational, open-label, and cohort studies as well as some case reports were also available. Furthermore, the methodologies of some SRs had limitations, and their conclusions can be subject to bias. Therefore, when multiple reviews reach the same conclusion, the conclusion should be explained carefully. Nevertheless, these are reported in detail in our results, and each conclusion can be assessed by the reader, including the limitations of the SRs. Another limitation was found in the selection of studies published solely in the English language, which limited the scope of the evidence mapping.

\section{Conclusion}

NP is a complex and refractory group of diseases. This evidence mapping could encourage clinical workers in the fields of pain, neurology, psychiatry, anesthesiology, and rehabilitation to pay more attention to individual patient characteristics and target the relevant brain regions. The small number of clinical trials in the area of non-M1 target therapy for NP is noteworthy, but the most important clinical issues have been covered as a result of evidence mapping. Evidence mapping is a useful and reliable method to identify currently available research as a suggestion for future research. In the future, more research effort is needed in order to highlight the optimal stimulation protocols and standardize all parameters to fill evidence gaps. More homogenous groups of participants should also be considered. Meanwhile, further efforts are needed to improve the methodological quality and reporting process of SRs.

\section{Data Availability}

The data generated in this study can be obtained in the supplementary materials provided.

\section{Conflicts of Interest}

The authors declare that the research was conducted in the absence of any commercial or financial relationships that could be construed as a potential conflict of interest.

\section{Authors' Contributions}

The study was designed by Yaning Zang, Yongni Zhang, and Yi Zhu. Screening studies and extraction data were collected by Yaning Zang and Xigui Lai under the guidance of Yongni Zhang. The data were analyzed by Yaning Zang and Yongni Zhang. Charting was done by Yaning Zang. The manuscript was drafted by Yaning Zang and Yongni Zhang. Yi Zhu reviewed the results. Shanshan Gu, Yujie Yang, Jiabao Guo, and Yi Zhu revised the manuscript for important intellectual content. All the authors approved the final version of the manuscript. Yaning Zang and Yongni Zhang contributed equally to this work.

\section{Acknowledgments}

This work was supported by the National Natural Science Foundation of China Project (No. 81860875).

\section{Supplementary Materials}

Supplementary Material 1: Database search strategies. Supplementary Material 2: SRs excluded. Supplementary Material 3: AMSTAR-2 assessment. Supplementary Material 4: PICOs' characteristics in the SRs. (Supplementary Materials)

\section{References}

[1] L. Colloca, T. Ludman, D. Bouhassira et al., "Neuropathic pain," Nature Reviews Disease Primers, vol. 3, no. 1, Article ID 17002, 2017.

[2] N. B. Finnerup, N. Attal, S. Haroutounian et al., "Pharmacotherapy for neuropathic pain in adults: a systematic review and meta-analysis," The Lancet Neurology, vol. 14, no. 2, pp. 162-173, 2015. 
[3] R. Baron, "Neuropathic pain: a clinical perspective," Handbook of Experimental Pharmacology, vol. 194, pp. 3-30, 2009.

[4] N. B. Finnerup, R. Kuner, and T. S. Jensen, "Neuropathic pain: from mechanisms to treatment," Physiological Reviews, vol. 101, no. 1, pp. 259-301, 2021.

[5] E. Cavalli, S. Mammana, and F. Nicoletti, "The neuropathic pain: an overview of the current treatment and future therapeutic approaches," International Journal of Immunopathology and Pharmacology, vol. 33, Article ID 2058738419838383, 2019.

[6] D. P. Kuffler, "Mechanisms for reducing neuropathic pain," Molecular Neurobiology, vol. 57, no. 1, pp. 67-87, 2020.

[7] K.-H. Kim, H.-J. Seo, S. Abdi, and B. Huh, "All about pain pharmacology: what pain physicians should know," The Korean Journal of Pain, vol. 33, no. 2, pp. 108-120, 2020.

[8] R. H. Dworkin, A. B. O'Connor, J. Kent et al., "Interventional management of neuropathic pain: NeuPSIG recommendations," Pain, vol. 154, no. 11, pp. 2249-2261, 2013.

[9] A. H. Iglesias, "Transcranial magnetic stimulation as treatment in multiple neurologic conditions," Current Neurology and Neuroscience Reports, vol. 20, no. 1, p. 1, 2020.

[10] A. Hirayama, Y. Saitoh, and H. Kishima, "Reduction of intractable deafferentation pain by navigation-guided repetitive transcranial magnetic stimulation of the primary motor cortex," Pain, vol. 122, no. 1-2, pp. 22-27, 2006.

[11] K. Bannister, J. Sachau, R. Baron, and A. H. Dickenson, "Neuropathic pain: mechanism-based therapeutics," Annual Review of Pharmacology and Toxicology, vol. 60, no. 1, pp. 257-274, 2020.

[12] M. J. Grant and A. Booth, "A typology of reviews: an analysis of 14 review types and associated methodologies," Health Information and Libraries Journal, vol. 26, no. 2, pp. 91-108, 2009.

[13] N. R. Haddaway, C. Bernes, B.-G. Jonsson, and K. Hedlund, "The benefits of systematic mapping to evidence-based environmental management," Ambio, vol. 45, no. 5, pp. 613-620, 2016.

[14] I. M. Miake-Lye, S. Hempel, R. Shanman, and P. G. Shekelle, "What is an evidence map? a systematic review of published evidence maps and their definitions, methods, and products," Systematic Reviews, vol. 5, no. 1, p. 28, 2016.

[15] P. Bragge, O. Clavisi, T. Turner, E. Tavender, A. Collie, and R. L. Gruen, "The global evidence mapping initiative: scoping research in broad topic areas," BMC Medical Research Methodology, vol. 11, no. 1, p. 92, 2011.

[16] M. Ballesteros, N. Montero, A. López-Pousa et al., "Evidence mapping based on systematic reviews of therapeutic interventions for gastrointestinal stromal tumors (GIST)," BMC Medical Research Methodology, vol. 17, no. 1, p. 135, 2017.

[17] V. Welch, T. E. Howe, and S. Marcus, "Protocol: health, social care and technological interventions to improve functional ability of older adults: evidence and gap map," Campbell Systematic Reviews, vol. 15, no. 4, Article ID e1054, 2019.

[18] M. Madera Anaya, J. V. A. Franco, and M. Ballesteros, "Evidence mapping and quality assessment of systematic reviews on therapeutic interventions for oral cancer," Cancer Management and Research, vol. 11, pp. 117-130, 2019.

[19] B. J. Shea, B. C. Reeves, G. Wells et al., “AMSTAR 2: a critical appraisal tool for systematic reviews that include randomised or non-randomised studies of healthcare interventions, or both," BMJ, vol. 358, Article ID j4008, 2017.

[20] M. A. Ahmed, E. S. Darwish, E. M. Khedr, Y. M. El serogy, and A. M. Ali, "Effects of low versus high frequencies of repetitive transcranial magnetic stimulation on cognitive function and cortical excitability in Alzheimer's dementia," Journal of Neurology, vol. 259, no. 1, pp. 83-92, 2012.

[21] K. Pacheco-Barrios, X. Meng, and F. Fregni, "Neuromodulation techniques in phantom limb pain: a systematic review and meta-analysis," Pain Medicine, vol. 21, no. 10, pp. 2310-2322, 2020.

[22] K. Gatzinsky, C. Bergh, A. Liljegren et al., "Repetitive transcranial magnetic stimulation of the primary motor cortex in management of chronic neuropathic pain: a systematic review," Scandinavian Journal of Pain, vol. 21, 2020.

[23] N. E. O'Connell, L. Marston, B. M. Wand, S. Spencer, and L. H. Desouza, "Non-invasive brain stimulation techniques for chronic pain," Cochrane Database of Systematic Reviews, vol. 9 , no. 4, 2018 .

[24] M. M. Klein, R. Treister, T. Raij et al., "Transcranial magnetic stimulation of the brain: guidelines for pain treatment research," Pain, vol. 156, no. 9, pp. 1601-1614, 2015.

[25] K. B. Jensen, C. Berna, M. L. Loggia, A. D. Wasan, R. R. Edwards, and R. L. Gollub, "The use of functional neuroimaging to evaluate psychological and other nonpharmacological treatments for clinical pain," Neuroscience Letters, vol. 520, no. 2, pp. 156-164, 2012.

[26] B. C. Ramger, K. A. Bader, S. P. Davies et al., "Effects of noninvasive brain stimulation on clinical pain intensity and experimental pain sensitivity among individuals with central post-stroke pain: a systematic review," Journal of Pain Research, vol. 12, pp. 3319-3329, 2019.

[27] A. Herrero Babiloni, S. Guay, D. Nixdorf, L. De Beaumont, and G. Lavigne, "Non-invasive brain stimulation in chronic orofacial pain: a systematic review," Journal of Pain Research, vol. 11, pp. 1445-1457, 2018.

[28] B. Yu, H. Qiu, J. Li, C. Zhong, and J. Li, "Noninvasive brain stimulation does not improve neuropathic pain in individuals with spinal cord injury," American Journal of Physical Medicine and Rehabilitation, vol. 99, no. 9, pp. 811-820, 2020.

[29] S. Yang and M. C. Chang, "Effect of repetitive transcranial magnetic stimulation on pain management: a systematic narrative review," Frontiers in Neurology, vol. 11, p. 114, 2020.

[30] X.-M. Xu, H. Luo, B.-B. Rong et al., "Nonpharmacological therapies for central poststroke pain," Medicine (Baltimore), vol. 99, no. 42, Article ID e22611, 2020.

[31] X. Moisset, B. Pereira, D. Ciampi de Andrade, D. Fontaine, M. Lantéri-Minet, and J. Mawet, "Neuromodulation techniques for acute and preventive migraine treatment: a systematic review and meta-analysis of randomized controlled trials," The Journal of Headache and Pain, vol. 21, no. 1, p. 142, 2020.

[32] X. Moisset, D. Bouhassira, J. Avez Couturier et al., "Pharmacological and non-pharmacological treatments for neuropathic pain: systematic review and French recommendations," Revue Neurologique, vol. 176, no. 5, pp. $325-352,2020$.

[33] A. Liampas, N. Velidakis, T. Georgiou et al., "Prevalence and management challenges in central post-stroke neuropathic pain: a systematic review and meta-analysis," Advances in Therapy, vol. 37, no. 7, pp. 3278-3291, 2020.

[34] A. Aamir, A. Girach, P. G. Sarrigiannis et al., "Repetitive magnetic stimulation for the management of peripheral neuropathic pain: a systematic review," Advances in Therapy, vol. 37, no. 3, pp. 998-1012, 2020.

[35] J. M. Stilling, O. Monchi, F. Amoozegar, and C. T. Debert, "Transcranial magnetic and direct current stimulation (TMS/ tDCS) for the treatment of headache: a systematic review," 
Headache: The Journal of Head and Face Pain, vol. 59, no. 3, pp. 339-357, 2019.

[36] P. Hamid, B. H. Malik, and M. L. Hussain, "Noninvasive transcranial magnetic stimulation (TMS) in chronic refractory pain: a systematic review," Cureus, vol. 11, no. 10, Article ID e6019, 2019.

[37] Y. Feng, B. Zhang, J. Zhang, and Y. Yin, "Effects of noninvasive brain stimulation on headache intensity and frequency of headache attacks in patients with migraine: a systematic review and meta-analysis," Headache: The Journal of Head and Face Pain, vol. 59, no. 9, pp. 1436-1447, 2019.

[38] L. Lan, X. Zhang, X. Li, X. Rong, and Y. Peng, "The efficacy of transcranial magnetic stimulation on migraine: a metaanalysis of randomized controlled trails," The Journal of Headache and Pain, vol. 18, no. 1, p. 86, 2017.

[39] H. Kumru, S. Albu, J. Vidal, and J. M. Tormos, "Effectiveness of repetitive trancranial or peripheral magnetic stimulation in neuropathic pain," Disability and Rehabilitation, vol. 39, no. 9, pp. 856-866, 2017.

[40] B. Goudra, D. Shah, G. Balu et al., "Repetitive transcranial magnetic stimulation in chronic pain: a meta-analysis," $A n$ esthesia: Essays and Researches, vol. 11, no. 3, pp. 751-757, 2017.

[41] L. Shirahige, L. Melo, F. Nogueira, S. Rocha, and K. MonteSilva, "Efficacy of noninvasive brain stimulation on pain control in migraine patients: a systematic review and metaanalysis," Headache: The Journal of Head and Face Pain, vol. 56, no. 10, pp. 1565-1596, 2016.

[42] J. J. Cragg, F. M. Warner, N. B. Finnerup et al., "Meta-analysis of placebo responses in central neuropathic pain," Pain, vol. 157, no. 3, pp. 530-540, 2016.

[43] C.-C. Chen, Y.-F. Chuang, A. C.-W. Huang, C.-K. Chen, and Y.-J. Chang, "The antalgic effects of non-invasive physical modalities on central post-stroke pain: a systematic review," Journal of Physical Therapy Science, vol. 28, no. 4, pp. 13681373, 2016.

[44] Y. Jin, G. Xing, and G. Li, "High frequency repetitive transcranial magnetic stimulation therapy for chronic neuropathic pain: a meta-analysis," Pain Physician, vol. 18, no. 6, pp. E1029-E1046, 2015.

[45] R. Galhardoni, G. S. Correia, and H. Araujo, "Repetitive transcranial magnetic stimulation in chronic pain: a review of the literature," Archives of Physical Medicine and Rehabilitation, vol. 96, no. 4 Suppl, pp. S156-S172, 2015.

[46] A. Leung, M. Donohue, R. Xu et al., "rTMS for suppressing neuropathic pain: a meta-analysis," The Journal of Pain, vol. 10, no. 12, pp. 1205-1216, 2009.

[47] A. V. Apkarian, M. N. Baliki, and P. Y. Geha, "Towards a theory of chronic pain," Progress in Neurobiology, vol. 87, no. 2, pp. 81-97, 2009.

[48] I. M. Miake-Lye, S. Mak, J. Lee et al., "Massage for pain: an evidence map," Journal of Alternative and Complementary Medicine, vol. 25, no. 5, pp. 475-502, 2019.

[49] R. Nardone, Y. Höller, P. B. Langthaler et al., "rTMS of the prefrontal cortex has analgesic effects on neuropathic pain in subjects with spinal cord injury," Spinal Cord, vol. 55, no. 1, pp. 20-25, 2017.

[50] R. A. A. de Oliveira, D. C. de Andrade, A. G. G. Machado, and M. J. Teixeira, "Central poststroke pain: somatosensory abnormalities and the presence of associated myofascial pain syndrome," BMC Neurology, vol. 12, no. 1, p. 89, 2012.

[51] S. Huang, S. L. Borgland, and G. W. Zamponi, "Dopaminergic modulation of pain signals in the medial prefrontal cortex: challenges and perspectives," Neuroscience Letters, vol. 702, pp. 71-76, 2019.

[52] C. Nuti, R. Peyron, and L. Garcia-Larrea, "Motor cortex stimulation for refractory neuropathic pain: four year outcome and predictors of efficacy," Pain, vol. 118, no. 1-2, pp. 43-52, 2005.

[53] A. K. Sahu, V. K. Sinha, and N. Goyal, "Effect of adjunctive intermittent theta-burst repetitive transcranial magnetic stimulation as a prophylactic treatment in migraine patients: a double-blind sham-controlled study," Indian Journal of Psychiatry, vol. 61, no. 2, pp. 139-145, 2019.

[54] J. Korzhova, I. Bakulin, D. Sinitsyn et al., "High-frequency repetitive transcranial magnetic stimulation and intermittent theta-burst stimulation for spasticity management in secondary progressive multiple sclerosis," European Journal of Neurology, vol. 26, no. 4, pp. 680-e44, 2019.

[55] R. Amin, T. Emara, S. Ashour et al., "The role of left prefrontal transcranial magnetic stimulation in episodic migraine prophylaxis," The Egyptian Journal of Neurology, Psychiatry and Neurosurgery, vol. 56, no. 1, p. 19, 2020.

[56] A. Leung, V. Metzger-Smith, Y. He et al., "Left dorsolateral prefrontal cortex rTMS in alleviating MTBI related headaches and depressive symptoms," Neuromodulation: Technology at the Neural Interface, vol. 21, no. 4, pp. 390-401, 2018.

[57] P. Lindholm, S. Lamusuo, T. Taiminen et al., "Right secondary somatosensory cortex-a promising novel target for the treatment of drug-resistant neuropathic orofacial pain with repetitive transcranial magnetic stimulation," Pain, vol. 156, no. 7, pp. 1276-1283, 2015.

[58] R. A. A. de Oliveira, D. C. de Andrade, M. Mendonça et al., "Repetitive transcranial magnetic stimulation of the left premotor/dorsolateral prefrontal cortex does not have analgesic effect on central poststroke pain," The Journal of Pain, vol. 15, no. 12, pp. 1271-1281, 2014.

[59] J. J. Borckardt, S. T. Reeves, P. Kotlowski et al., "Fast left prefrontal rTMS reduces post-gastric bypass surgery pain: findings from a large-scale, double-blind, sham-controlled clinical trial," Brain Stimulation, vol. 7, no. 1, pp. 42-48, 2014.

[60] U. K. Misra, J. Kalita, and S. K. Bhoi, "High-rate repetitive transcranial magnetic stimulation in migraine prophylaxis: a randomized, placebo-controlled study," Journal of Neurology, vol. 260, no. 11, pp. 2793-2801, 2013.

[61] E. M. Khedr, M. A. Ahmed, E. A. M. Alkady, M. G. Mostafa, and H. G. Said, "Therapeutic effects of peripheral magnetic stimulation on traumatic brachial plexopathy: clinical and neurophysiological study," Neurophysiologie Clinique/Clinical Neurophysiology, vol. 42, no. 3, pp. 111-118, 2012. 